\title{
Three Dimensional Laser Data Inspector Based on Data Processing
}

\author{
Pankaj Saraswat
}

\begin{abstract}
The most accurate exploration method of acquiring three-dimensional remote sensing knowledge is remotely detected ' 3D Laser Scan' software. Laser scanning system enhances the capability to regulate spatial attributes of any three-dimensional object. The perused object is further pictured by expert software as a group of points. The implementation has been much favored in several areas because of its simplicity and accuracy. However, the information volume is fairly large and calls for unique, costly patented programs for transformation. In this research, a LiDAR Read and excerpt(LiRE) python-based program with different python modules is introduced. It is helpful for reading, viewing, filtering and exporting LAS information in different sizes. It also utilizes unsupervised and supervised techniques in order to classify information according to training data in separate classes.
\end{abstract}

Keywords: 3D Laser Scan, LiDAR, Data Processing, LiRE, Python.

\section{INTRODUCTION}

The concept of "laser imaging" is used in contemporary engineering to describe two associated but distinct concepts. In stereo-thallic devices, in rapid prototyping, in material processing machines, in laser graveling devices, in ophthalmologic presbyopia-processing laser applications. The monitored rotations of laser pulses accompanied by range measurements in every path of motion have more particular significance. This technique, which is often introduced to as 3D image processing, is used to quickly record the forms of artifacts, structures, and landscapes. Usually, the $3 \mathrm{D}$ scanner objective is to generate a geometrical point cloud on the topic ground. These marks can then be useful for extrapolating of the topic. Almost all of the current research has drawn up designs and requirements for information ranking restricted to particular areas that require changes in designs. Moreover, the surveys employ software engineering instruments, such as matrix laboratory, $\mathrm{C}, \mathrm{C}++$, etc. for the creation and implementation of information identification systems. Data processing is now commonly used to define model content using teaching content and to address fresh entry data classifications that decide the characteristics of fresh entry data using such model data. The traditional ranking model includes Detailed Tree, Rule Set, Bayesian Network, Support Vector Machine, and Neurons. The precision of ranking depends on how the user selects designs and class kinds in the dataset. A pythondriven program for 3D Laser Scan Information management was formed in this research, LiDAR Read \& Export (Lire). The software then reads and views the LAS documents and exports them in a discrete comma or tab-detailed folder and enters information directly. In addition, information can be

Revised Manuscript Received on September 14, 2019.

Pankaj Saraswat, Department of Computer Science and Engineering, Sanskriti University, Uttar Pradesh, India.(Email: sanpubip@gmail.com) processed on the median by region, height and smaller or greater, and visualized in 2D, 3D and ground charts. Finally, it is possible to classify information straight on the basis of monitored or training-based ranking methods centered on controlled data processing.

\section{RELATED WORK}

In order to precisely reconstruct the particles of different objects and sites, 3D laser scan was produced throughout the last half of the 20th century. In studies and layout, the technology is particularly useful. The very first 3D imaging system was developed in the 1960s. The premature scanners were able to execute this job through lamps, sensors, and projectors. Due to restrictions on the machinery, the scanning of the items often requires a ton of moment and effort. They were substituted after 1985 by scanners which would record a specified layer using white light, lasers, and shadows. In the 1980s a touch test was created for the toolmaking sector. This allowed at least the creation of a precise model, but it was so delayed. The thought was that if someone could develop a scheme that caught the same quantity of information but made its implementation more efficient at a greater velocity. Analysts have therefore managed to develop optical innovation. It was quicker to use light than a physical test. This also enables the scan of soft particles that are in danger of prodding. An instance of a 3D touch detector is a CMM (Coordinate Measurement Machine). A Coordinate Measuring Machine (CMM) is a modeling tool for recording an object's physical geometry. This device can be regulated directly by an assistant or regulated by the laptop. A sample connected to the fifth shifting arm of the device defines measures. Samples may include a mechanical, optical, flashlight or blacklight. In 1994, 3D Scanners started REPLICA, allowing quick and very precise object processing. In laser ribbon screening REPLICA labeled severe advancement in laser stripe scanning. While Cyberware developed own high-intake scanners, some of whom also were capable of capturing color, real three-dimensional imaging-with such velocity and precision levels-stayed unknown amid this advancement. Digibotics was one firm that introduced a four-axis computer, which was able to provide a completely 3D template from a given test, but relied on the electron tip rather than the beam strip, and therefore delayed. Nor was he able to digitize the shade surface with the six degrees of freedom needed for the whole surface of an object. 
A researcher in paper [1] proposed a differential threedimensional scanning technique, in which with each iteration the developer produces a three-dimensional model and print that model using a three-dimensional printer and then some editing is done on the model, again scanned it back to amend the deviations. But it produces data which is complex to handle and understand also requires costly branded software.

Researches had been carried out in Korea by employing various methods and algorithms of the fruitful application of three dimensional scannings. Another paper [2] employed Local Indicators of Spatial Association (LISA) measurements deviations for recognizing buildings. Correspondingly, [3] suggested a new algorithm for mining out artificial arrangements present in three-dimensional cloud points which are implemented on the basis of red, green, blue color space and Houge transformation. Another paper [4] clarified some airborne laser scanning points over labeling and scan line adjacency algorithm. Another research paper [5] proposed various methods for clustering the data features. Yet another paper [6] presented research in which discloses about the title depended on the classification of topography by airborne data extraction of building the skeleton.

\section{PROPOSED SYSTEM}

The proposed method is coded in a python programming language. Python has an open source module. Anaconda software was used for the full science suite to ameliorate efforts in connecting various modules. The information on all program features is given in Table 1. Five menus of this program: LAS file opener and exports, divided or panel documents, 2D and 3D plot view, filter for scanning and producing fresh documents, classified data processing and finally data. Laser folder is accessed by LibLAS and maintained with NumPy and SciPy components, while matplotlib and mayavi applications are used to visualize the mas folder. Science-learning and orange modules are alternatively used to make the not monitored and supervised categorization.

Table 1 Name of section and description of tasks.

\begin{tabular}{|c|c|c|}
\hline Menu & Sub Menu & Function \\
\hline \multirow{4}{*}{ File } & New & Reset file selection \\
\hline & Open & $\begin{array}{l}\text { Open las file and training } \\
\text { input }\end{array}$ \\
\hline & Export & Export in las, txt, tab file \\
\hline & Quit & \\
\hline \multirow{8}{*}{ View } & $\begin{array}{l}\text { Feature Boundary } \\
\text { Line }\end{array}$ & \multirow{8}{*}{$\begin{array}{l}\text { Visualize boundary, } 2 \mathrm{D} \\
\text { and } 3 \mathrm{D} \text { plots }\end{array}$} \\
\hline & RGB Scatter Plots & \\
\hline & 2D Scatter Plot & \\
\hline & 2D TIN Plot & \\
\hline & 2D Contour Plot & \\
\hline & 3D Scatter Plot & \\
\hline & 3D TIN Plot & \\
\hline & 3D Overview & \\
\hline \multirow{6}{*}{ Filter } & Area(XY Range) & \multirow{6}{*}{$\begin{array}{l}\text { Filter } 3 \mathrm{D} \text { points based on } \\
\text { the area range, elevation }\end{array}$} \\
\hline & Elevation & \\
\hline & Intensity & \\
\hline & LowFeature Points & \\
\hline & HighFeature Points & \\
\hline & Density & \\
\hline \multirow{6}{*}{$\begin{array}{l}\text { Data } \\
\text { Mining }\end{array}$} & $\begin{array}{l}\text { Unsupervised } \\
\text { KMeans }\end{array}$ & Using $\mathrm{K}$-means clusters \\
\hline & Classification Tree & \multirow{5}{*}{$\begin{array}{l}\text { Requires training input } \\
\text { data in file menu }\end{array}$} \\
\hline & K-Nearest & \\
\hline & Neighbours & \\
\hline & Naive Bayesian & \\
\hline & $\begin{array}{l}\text { Support Vector } \\
\text { Machine }\end{array}$ & \\
\hline
\end{tabular}

File Menu LiDAR mas documents were viewed and transported in a txt file with distinct modules, xls for other GIS/ CAD applications, information processing programs such as WEKa, SPSS, etc.

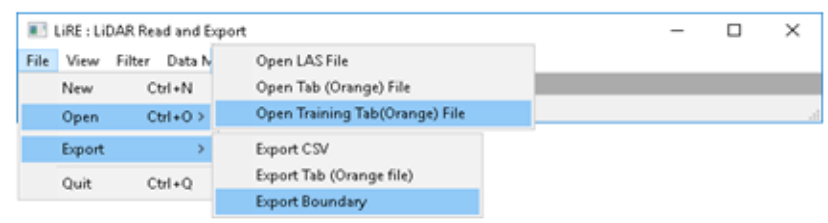

\section{Figure 1 LiRE GUI with Menu File and Submenu}

In every geospatial data, data visualization is very essential. The View panel in Lire allows the screen region and RGB dispersions to be defined. Data can be traced by the operator on 2D X-Y and 3D X - Y-Z planes. Also, depending upon altitude, TIN and contour can be regarded. The 3D panorama is focused on the entry data's height and strength.

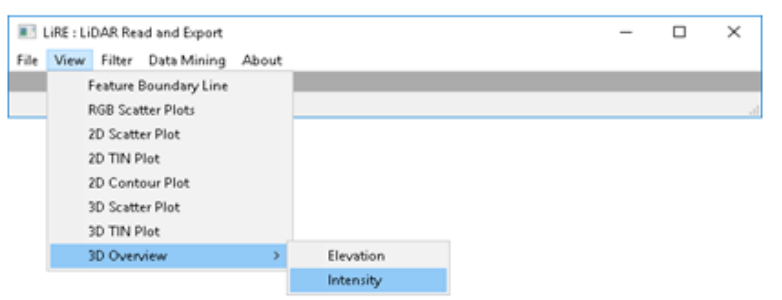

Figure 2 View menu submenus.

Borderline removal in civil engineering is of great importance as it can be readily removed after ranking or screening with characteristics like highways or houses. The Convex Hull technique is used for frontier removal in the Boundary line feature module. The polygon with rectangular corners is formed in order to only include all the values given, as shown in Figure 3.

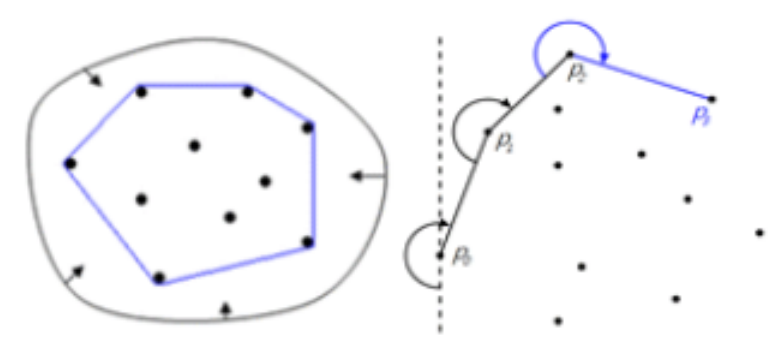

Figure 3 convex closure

As the pure $3 \mathrm{D}$ camera test information is very thick and not always all measurements are required. For efficient and effective information implementation, filtering is therefore crucial. The submenus of the scheduling alternatives are displayed in Figure 4. The surface-depth filter is designed to cut the rectangular subset, while an input-based depth and strength can be used to filter. Maximum and minimum feature scores are segregated from the average valuation, which is highly helpful in selecting offsets or individual

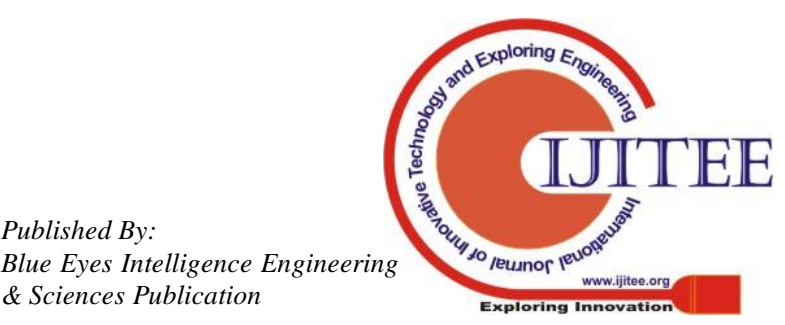


items such as roads. The Density choice uniformly monitors the information depending on the supplied 75,50 and $25 \%$ of the entry in the fresh output folder.

In the data processing panel, the ranking of 3D laser scanned information as shown by figure 5 is not monitored or monitored. Unattended classifications use K-means for forming and exporting clusters in CSV or tab formats; while the supervised Classification Trees, Naive Bayesian and Support Vector Machine methods require input data from the File menu based on the definition of new modes for each point.

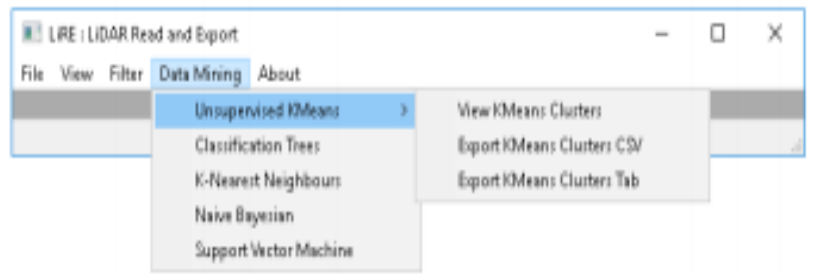

Figure 4 filter the program's submenus.

\section{RESULT}

In the Gurugram road region with highways, vegetation, concrete towers, road lamps, and true world 3D Laser Scan information was produced to monitor the Program's output. The information is available in Las configuration and was first launched to view the images in Figure 5 in the LIRE program. The picture on the left displays only bounding charts in blue, while the picture on the right is 3D where the path and corner are visible. Due to the fact that the information is very thick, the filter features were also screened, and all of that information appears to be working well.

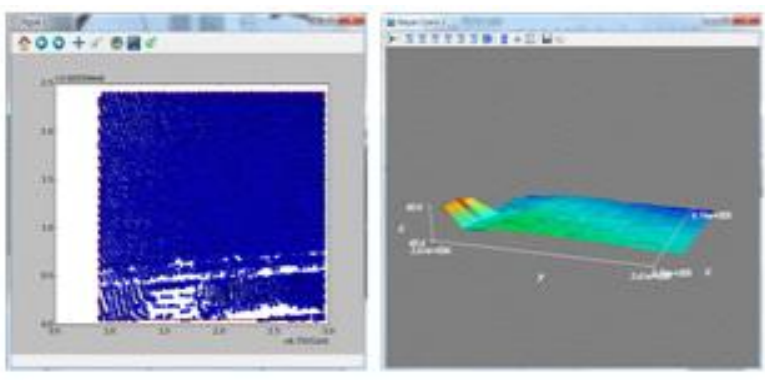

Figure 5 Data on the 2D and 3D road part plots.

Since the information is very thick, the filtering features were checked as well and all of them seemed to operate correctly. Figure 6 demonstrates the 75 percent density processing and Figure 7 demonstrates elevated and small information scores from the screen median.
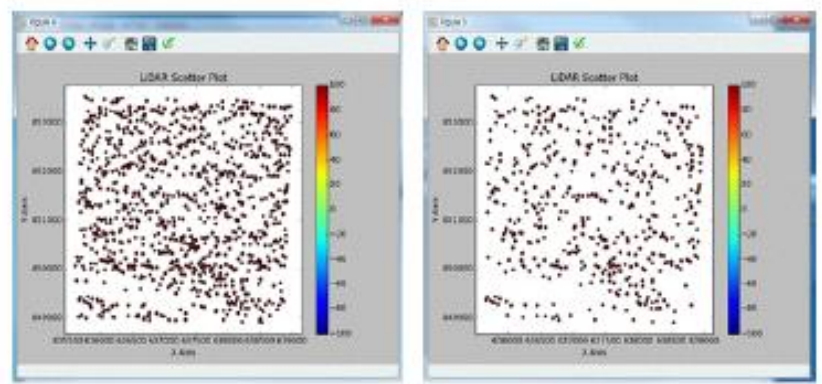

Figure 6 Filter output at $75 \%$ density.

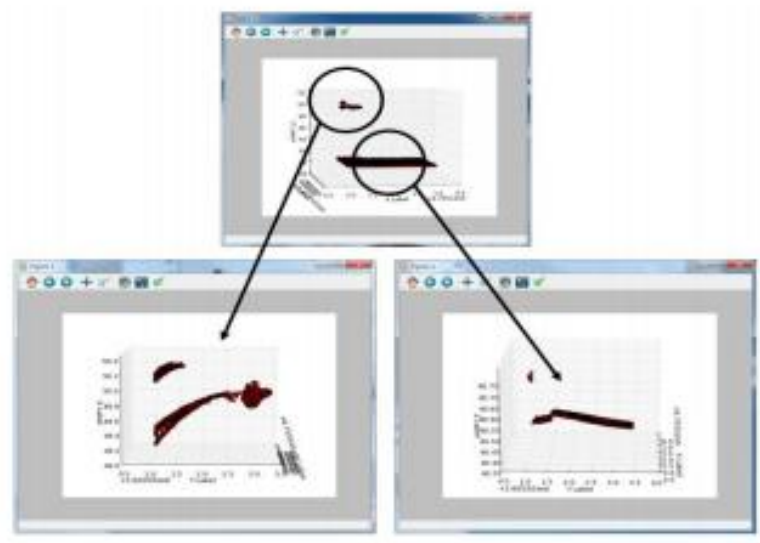

Figure 7 High and low-function point filtering.

Figure 8 and Figure 9 portrays the precision of ranking with complete characteristics and chosen characteristics. The precision of the ranking appears to be enhanced with the chosen characteristics.

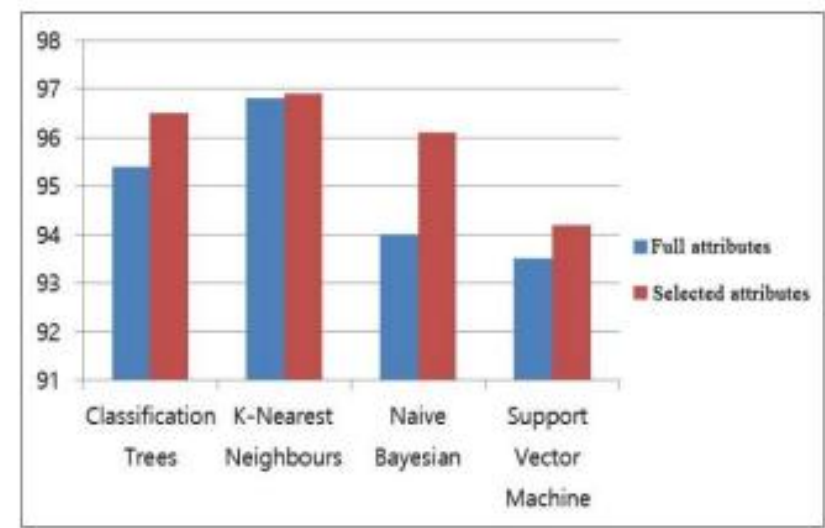

Figure 8 Precise description of the test controlled depending on the pairs of characteristics.

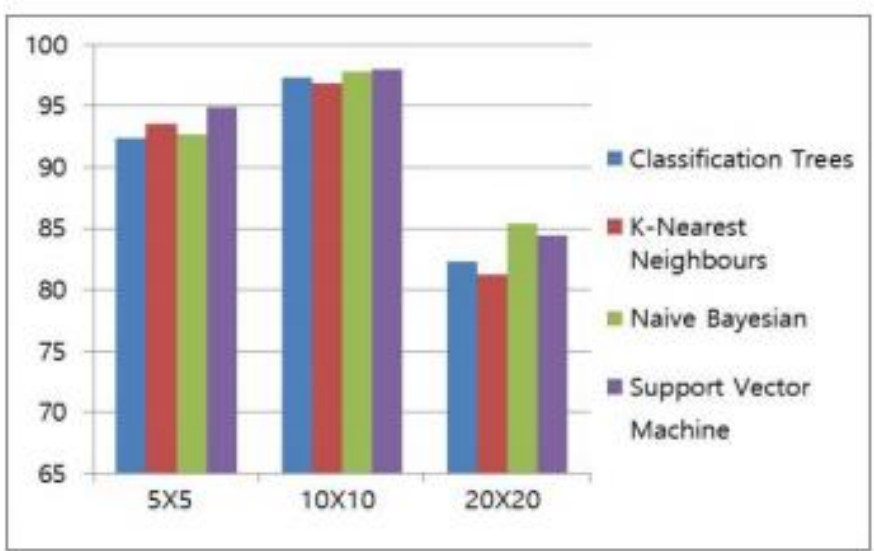

Figure 9 Classification accuracy of supervised classification 


\section{CONCLUSION}

3D Laser Scan Data management in python setting has been developed using different modules in this paper. The last two hundred years, laser scanning engineering has evolved and matured into a major geographic data procurement technology. There is a variety of openly available accessible tools with different capacities. In several specific areas of the survey, such as topography, the habitat, and industry, premium quality information generated by satellite scanners are now used. The enhanced program proposed in this paper is able to read, export, view, filter and categorize laser documents. There is an increase in the amount of information for routing and controlled information evaluation have also been screened and implemented. Filtering information depending on region, height, strength, and thickness may decrease the expense of calculation and transport and may contribute to stronger outcomes for ranking. In the future, the program will promote new types of LiDAR, scan them more effectively and ranks them with more precision.

\section{REFERENCES}

1. A. Hattab, I. Gonsher, D. Moreno, and G. Taubin, "Differential 3d scanning," IEEE Computer Graphics and Applications, vol. 38, no. 3, pp. 43-51, 2017.

2. Byun, Y.; Lee, J.; Son, J.; Yu, K. A Study on Building Extraction from LiDAR Data using LISA. Journal of the Korean Society of Surveying, Geodesy, Photogrammetry and Cartography 2006, 24, 335-341.

3. Kim, E.; Wie, G.; Cho, H.; Yang, I. A Study for Forest Research using Airborne Laser Scanning. Journal of the Korean Society of Surveying, Geodesy, Photogrammetry and Cartography 2010, 28, 299-304.

4. Lee, J.H.; Yeom, J.H.; Kim, Y.I. Filtering Airborne Laser Scanning Data by Utilizing Adjacency Based on Scan Line. Journal of the Korean Society of Surveying, Geodesy, Photogrammetry and Cartography 2011, 29, 359-365.

5. Choi, S.P.; Cho, J.H.; Kim, Y.; Kim, J.S. Classification of Terrestrial LiDAR Data using Factor and Cluster Analysis. Journal of the Korean Society for Geo-spatial Information Science 2011, 19, 139-144.

6. Kim, N.; Kim, Y. A Building Outline Extraction Scheme using Tile-Based Topographical Classification from Aerial LiDAR Data and Building Tile's Airborne Image. Journal of KIISE: Computing Practices and Letters 2012, 18, 916-920.

7. Yang, I.T.; Acharya, T.D.; Lee, D.H.; Shin, M.S. Reverse Engineering of a Bobsleigh Structure using LiDAR. International Journal of Applied Engineering Research 2017, 12, 976-980.

8. Hall, M.; Frank, E.; Holmes, G.; Pfahringer, B.; Reutemann, P.; Witten, I.H. The WEKA Data Processing Software: An Update. SIGKDD Explor.Newsl. 2009, 11, $10-18$.

9. Lee, S.; Lee, H.; Sung, C.; Park, C.; Cho, W.; Kim, Y. A Topographical Classifier Development Support System Cooperating with Data Processing Tool WEKA from Airborne LiDAR Data. Journal of the Korean Society of Surveying, Geodesy, Photogrammetry and Cartography 2010, 28, 133-142.

10. I. A. Anderson, T. A. Gisby, T. G. McKay, B. M. O'Brien, and E. P. Calius, "Multi-functional dielectric elastomer artificial muscles for soft and smart machines,' Journal of Applied Physics, vol. 112, no. 4, p. 041101, 2012.
11. B. Alsadik, M. Gerke, and G. Vosselman, "Visibility analysis of point cloud in close range photogrammetry," ISPRS Annals of the Photogrammetry, Remote Sensing and Spatial Information Sciences, vol. 2, no. 5, p. 9, 2014.

12. D. Feurer, M. A. El Maaoui, O. Planchon, M. R. Boussema, and M. Pierrot-Deseilligny, "Kite-borne photogrammetry for decimetric 3d mapping of several square kilometres areas," in Geoscience and Remote Sensing Symposium (IGARSS), 2015 IEEE International. IEEE, 2015, pp. 3858-3861.

13. "Openmp," http://www.openmp.org/, accessed: 2017 12-21.

14. F. Buffa, A. Pinna, and G. Sanna, "A simulation tool assisting the design of a close range photogrammetry system for the Sardinia radio telescope.” ISPRS Annals of Photogrammetry, Remote Sensing \& Spatial Information Sciences, vol. 3, no. 5, 2016.

15. " $3 \mathrm{dm}$ https://www.adamtech.com.au/3dm/Analyst.html, accessed: 2017-12-12.

16. R. Blomley and M. Weinmann, "Using multi-scale features for the $3 \mathrm{~d}$ semantic labeling of airborne laser scanning data," ISPRS Annals of the Photogrammetry, Remote Sensing and Spatial Information Sciences, Volume IV-2/W4, 2017.

17. Palomer, Albert, et al. "3D laser scanner for underwater manipulation." Sensors 18.4 (2018): 1086.

18. Stutz, David, and Andreas Geiger. "Learning 3d shape completion from laser scan data with weak supervision." Proceedings of the IEEE Conference on Computer Vision and Pattern Recognition. 2018.

19. Behley, Jens, and Cyrill Stachniss. "Efficient SurfelBased SLAM using 3D Laser Range Data in Urban Environments." Robotics: Science and Systems. 2018.

20. Cheng, Liang, et al. "Registration of laser scanning point clouds: A review." Sensors 18.5 (2018): 1641. 